\title{
Correntropy: Implications of NonGaussianity for the Moment Expansion and Deconvolution
}

\author{
Z. Yang ${ }^{\mathrm{a}}$, A. T. Walden ${ }^{\mathrm{a}, *}$, E. J. McCoy ${ }^{\mathrm{a}}$ \\ ${ }^{a}$ Department of Mathematics, Imperial College London, 180 Queen's Gate, London SW7 \\ 2BZ, $U K$
}

\begin{abstract}
The recently introduced correntropy function is an interesting and useful similarity measure between two random variables which has found myriad applications in signal processing. A series expansion for correntropy in terms of higher-order moments of the difference between the two random variables has been used to try to explain its statistical properties for uses such as deconvolution. We examine the existence and form of this expansion, showing that it may be divergent, e.g., when the difference has the Laplace distribution, and give sufficient conditions for its existence for differently characterized sub-Gaussian distributions. The contribution of the higher-order moments can be quite surprising, depending on the size of the Gaussian kernel in the definition of the correntropy. In the blind deconvolution setting we demonstrate that statistical exchangeability explains the existence of sub-optimal minima in the correntropy cost surface and show how the positions of these minima are controlled by the size of the Gaussian kernel.
\end{abstract}

Key words: Blind Deconvolution, Correntropy, Sub-Gaussian Distribution, Super-Gaussian Distribution.

\footnotetext{
*Corresponding author. Tel: (0)20 7594 8524; Fax: (0)20 7594 8517; e-mail: a.walden@imperial.ac.uk
} 


\section{Introduction}

Recently there has been a surge of interest in the 'correntropy' function which is a generalized similarity measure between two random variables $X$ and $Y$ defined by

$$
V\left(X, Y ; \sigma_{K}\right)=E\left\{K_{\sigma_{K}}(X-Y)\right\}
$$

where $E\{\cdot\}$ denotes expected value, and $K_{\sigma_{K}}(\cdot)$ is the Gaussian kernel

$$
K_{\sigma_{K}}(z)=\frac{1}{\sigma_{K} \sqrt{2 \pi}} \mathrm{e}^{-z^{2} / 2 \sigma_{K}^{2}},
$$

$\sigma_{K}>0$ being the kernel size. (Another continuous, symmetric, non-negative definite kernel could be chosen, but the Gaussian is almost invariably used in practice. The random variables could be vector-valued, but for simplicity we only consider scalar variables.) Possible applications are increased by the fact that $X$ and $Y$ can be elements of two stochastic processes so that correntropy can reflect both temporal (correlation) and distributional structures in the processes.

Correntropy was introduced by Santamaria et al. [23] who suggested an application to blind deconvolution. Subsequent work reported in this journal has used correntropy for blind source separation [15] and as a measure for determining nonlinear dynamics [9]. A correntropy-based minimum average correlation energy (MACE) filter is given in [11]. Further discussions on correntropy can be found in [17] and [20].

Let $Z=X-Y$. In what follows we shall write $V\left(Z ; \sigma_{K}\right)$ more simply as $V$ and $K_{\sigma_{K}}(Z)$ as $K(Z)$. Using a series expansion for the Gaussian kernel $K(\cdot)$,

$$
\begin{aligned}
V & =E\{K(Z)\} \\
& =\frac{1}{\sigma_{K} \sqrt{2 \pi}} \int_{-\infty}^{\infty} \sum_{n=0}^{\infty} \frac{(-1)^{n}}{n !}\left[\frac{z^{2}}{2 \sigma_{K}^{2}}\right]^{n} f(z) \mathrm{d} z,
\end{aligned}
$$

where $f(\cdot)$ is the probability density function (PDF) of $Z$. The correntropy is written in $[9,23,15,11]$ as

$$
V=\frac{1}{\sigma_{K} \sqrt{2 \pi}} \sum_{n=0}^{\infty} \frac{(-1)^{n}}{2^{n} \sigma_{K}^{2 n} n !} E\left\{Z^{2 n}\right\},
$$


which assumes the interchange of integration and summation. Equation (4) was interpreted in $[9,15,11]$ to mean that correntropy thus involves higher-order even moments of $Z$. Indeed [11, p. 874] "the kernel size $\sigma_{K}$ controls the emphasis of the higher order moments with respect to the second, since the higher order terms of the expansion decay faster for larger $\sigma_{K}$. As $\sigma_{K}$ increases, the high order moments decay and the second-order moment tends to dominate." The expansion (4) is clearly important in trying to understand the statistical properties of correntropy, but to-date has not been carefully scrutinized or justified. It is well-recognized [17] that the kernel size "should be selected according to the application." The random variable $Z$ will have a particular distribution and a standard deviation which we denote throughout by $\sigma$. In Sections 2 and 3 of this paper we look at the effect the ratio $\sigma_{K} / \sigma$ and the distribution of $Z$ have on our interpretation of (4). It is important to appreciate that it is not necessary for the expansion (4) to exist for correntropy to exist. However (4) has been widely publicized in connection with correntropy and it is timely to examine its justification. In Section 5 we return to the initial application of correntropy, namely blind deconvolution [23], to further illustrate the effects of the size of $\sigma_{K}$, firstly on the moments contributing to (4), and secondly on the minima of the correntropy cost surface.

\section{Correntropy and its expansion in terms of moments}

In the following discussion we shall use the generalized Gaussian distribution (GGD) as a convenient parametric form for examining nonGaussianity in the correntropy setting. The zero-mean and symmetric GGD has the density function:

$$
f(z)=\frac{\alpha A(\alpha, \sigma)}{2 \Gamma(1 / \alpha)} \mathrm{e}^{-[A(\alpha, \sigma)|z|]^{\alpha}}, \quad|z|<\infty,
$$

with $A(\alpha, \sigma)=\left\{\Gamma(3 / \alpha) /\left[\sigma^{2} \Gamma(1 / \alpha)\right]\right\}^{1 / 2}$, where $\alpha$ is the shape parameter and $\sigma>0$ is the standard deviation. $\alpha=1$ gives the Laplace distribution, $\alpha=2$ is the Gaussian and as $\alpha \rightarrow \infty$ the uniform distribution is derived. With (4) in 

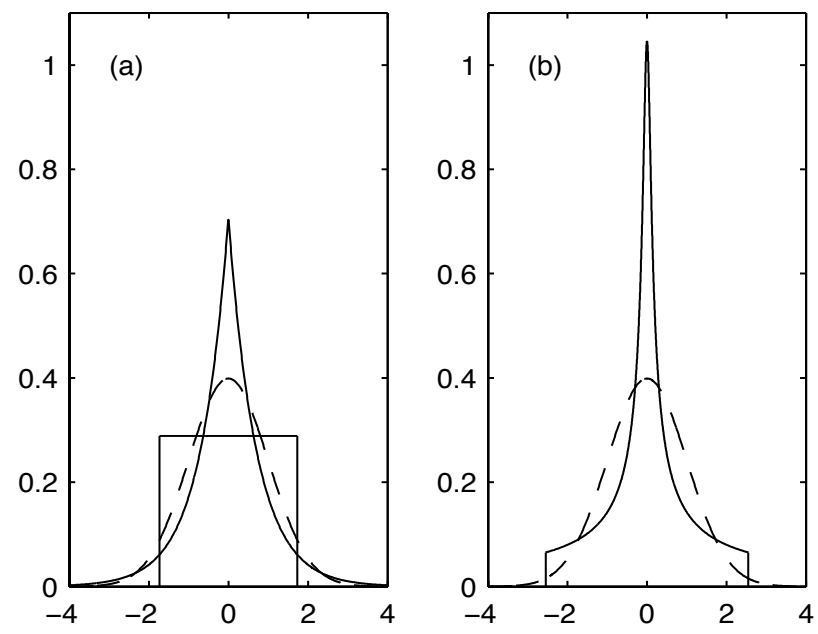

Figure 1: Distributions with standard deviations of unity. (a) Laplace (peaked), Gaussian (dashed) and uniform (flat). (b) Tukey's lambda distribution (solid line) and Gaussian (dashed).

mind, we note that for the GGD,

$$
E\left\{Z^{2 n}\right\}=\frac{\Gamma^{n-1}(1 / \alpha) \Gamma([2 n+1] / \alpha)}{\Gamma^{n}(3 / \alpha)} \sigma^{2 n} .
$$

Fig. 1(a) plots the Laplace, Gaussian and uniform distributions, all with standard deviation $\sigma$ of unity. The shape parameter determines the peakedness at the origin and long-tailedness of the distribution relative to the Gaussian.

\subsection{A Divergent Expansion}

Suppose $Z$ has a Laplace distribution specified by (5) with $\alpha=1$; the PDF takes the simple form $f(z)=(1 /[\sigma \sqrt{2}]) \mathrm{e}^{-|z| \sqrt{2} / \sigma}$, and from $(6), E\left\{Z^{2 n}\right\}=$ $(2 n) ! \sigma^{2 n} / 2^{n}$. Then (4) gives

$$
V=\frac{1}{\sigma_{K} \sqrt{2 \pi}} \sum_{n=0}^{\infty} \frac{(-1)^{n}(2 n) !}{n !}\left[\frac{\sigma}{2 \sigma_{K}}\right]^{2 n}
$$

Let $u_{n}$ denote the $n$th term of the sum, then $\left|u_{n+1} / u_{n}\right|=(n+1 / 2)\left(\sigma^{2} / \sigma_{K}^{2}\right) \rightarrow \infty$ as $n \rightarrow \infty$, so the sum is divergent by the ratio test. While the ratio test shows that the sum in (7) is divergent, it tells us nothing about the nature of the 
divergence. We will now show that (7) is in fact an asymptotic series expansion [7]; an asymptotic series typically occurs when the mathematically incorrect procedure of exchanging the order of summation and integration is carried out. To show this we make use of the form of $K(\cdot)$ directly,

$$
\begin{aligned}
V & =E\{K(Z)\} \\
& =\frac{1}{2 \sigma \sigma_{K} \sqrt{\pi}} \int_{-\infty}^{\infty} \mathrm{e}^{-z^{2} /\left(2 \sigma_{K}^{2}\right)} \mathrm{e}^{-|z| \sqrt{2} / \sigma} \mathrm{d} z \\
& =\frac{1}{\sigma \sigma_{K} \sqrt{\pi}} \int_{0}^{\infty} \mathrm{e}^{-\left[z+\left(\sigma_{K}^{2} \sqrt{2} / \sigma\right)\right]^{2} /\left(2 \sigma_{K}^{2}\right)+\sigma_{K}^{2} / \sigma^{2}} \mathrm{~d} z \\
& =\frac{\mathrm{e}^{\sigma_{K}^{2} / \sigma^{2}}}{\sigma \sigma_{K} \sqrt{\pi}} \int_{\sigma_{K} / \sigma}^{\infty} \mathrm{e}^{-y^{2}} \sigma_{K} \sqrt{2} \mathrm{~d} y .
\end{aligned}
$$

Now the complementary error function is defined as $\operatorname{erfc}(z)=(2 / \sqrt{\pi}) \int_{z}^{\infty} \mathrm{e}^{-y^{2}} \mathrm{~d} y$, so we can write

$$
V=\frac{\mathrm{e}^{\sigma_{K}^{2} / \sigma^{2}}}{\sigma \sqrt{ } 2} \operatorname{erfc}\left(\sigma_{K} / \sigma\right)
$$

But $\operatorname{erfc}(\cdot)$ has an asymptotic series expansion $[1,7.1 .23]$

$$
\sqrt{\pi} z \mathrm{e}^{z^{2}} \operatorname{erfc}(z)=\sum_{n=0}^{\infty} \frac{(-1)^{n}(2 n) !}{n !(2 z)^{2 n}} .
$$

Putting $z=\sigma_{K} / \sigma$ and substituting in (8) we obtain (7).

The asymptotic series has an interesting property: even though (9) is a divergent series, if it is truncated at its smallest magnitude term, retaining only its first few terms, it will give an excellent approximation to the function. In fact truncation at $n^{\prime} \doteq z^{2}$, is appropriate for the erfc function [7, p. 2], so we get $n^{\prime} \doteq \sigma_{K}^{2} / \sigma^{2}$, the ratio of the variance of the Gaussian kernel to the variance of the Laplace-distributed random variable $Z$. This is illustrated in Fig. 2. The plots in the left column show just the first few terms in the expansion (4), while the right column shows a larger number of terms with big changes in the scaling on the y-axes of the plots. Take the case $\sigma_{K}=3, \sigma=1$, so $n^{\prime} \doteq 9$. Using in fact only 7 terms in (4), plot (a), there is agreement with (8). However, after about term 20 oscillations start to occur in the terms of (4), plot (b), and these grow and grow. Now consider $\sigma_{K}=1, \sigma=1$, for which we would not expect 

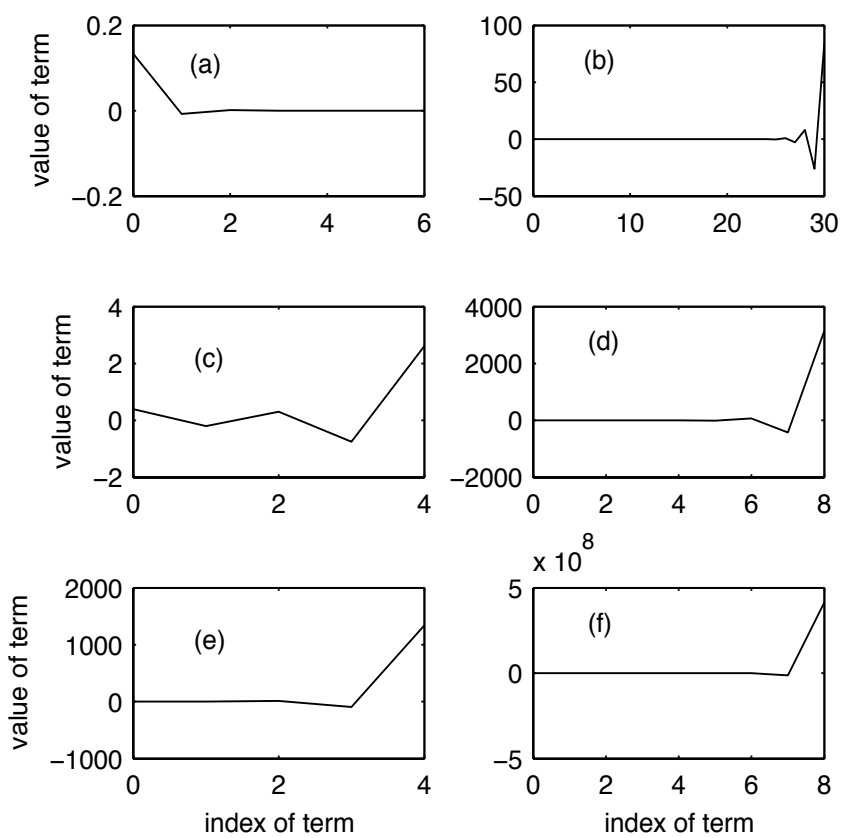

Figure 2: Terms in the expansion (4) for a Laplace distribution for $Z,(\sigma=1)$ and $\sigma_{K}=3$ for (a) and (b), $\sigma_{K}=1$ for (c) and (d), and $\sigma_{K}=0.5$ for (e) and (f).

the asymptotic expansion to work. This is shown to be the case in plots (c) and (d) of Fig. 2; even more extreme oscillatory behaviour is seen in plots (e) and (f) of Fig. 2 for $\sigma_{K}=0.5, \sigma=1$.

\subsection{A Sufficient Condition for Convergence}

Having seen an example where the expansion (4) is divergent, we now consider conditions for its convergence. Let us write (3) as

$$
V=\frac{1}{\sigma_{K} \sqrt{2 \pi}} \int_{-\infty}^{\infty} \lim _{N \rightarrow \infty} \sum_{n=0}^{N} \frac{(-1)^{n}}{n !}\left[\frac{z^{2}}{2 \sigma_{K}^{2}}\right]^{n} f(z) \mathrm{d} z .
$$

If we define $g_{N}(z)=\sum_{n=0}^{N} \frac{(-1)^{n}}{n !}\left[\frac{z^{2}}{2 \sigma_{K}^{2}}\right]^{n} f(z)$ then this can be written as

$$
V=\frac{1}{\sigma_{K} \sqrt{2 \pi}} \int_{-\infty}^{\infty} \lim _{N \rightarrow \infty} g_{N}(z) \mathrm{d} z .
$$


Now $\lim _{N \rightarrow \infty} g_{N}(z)=g(z)=\sum_{n=0}^{\infty} \frac{(-1)^{n}}{n !}\left[\frac{z^{2}}{2 \sigma_{K}^{2}}\right]^{n} f(z)$. and so by the Dominated Convergence Theorem, (e.g., [18, p. 538]), if , for all $N \in \mathbb{N}$,

$$
\left|g_{N}(z)\right| \leq G(z) \text { and } \int_{-\infty}^{\infty} G(z) \mathrm{d} z<\infty
$$

then $g(z)$ is integrable and $V$ in (10) becomes

$$
V=\frac{1}{\sigma_{K} \sqrt{2 \pi}} \lim _{N \rightarrow \infty} \sum_{n=0}^{N} \int_{-\infty}^{\infty} \frac{(-1)^{n}}{n !}\left[\frac{z^{2}}{2 \sigma_{K}^{2}}\right]^{n} f(z) \mathrm{d} z
$$

and so (4) is obtained. But

$$
\begin{aligned}
\left|g_{N}(z)\right| & \leq \sum_{n=0}^{N}\left|\frac{(-1)^{n}}{n !}\left[\frac{z^{2}}{2 \sigma_{K}^{2}}\right]^{n} f(z)\right|=\sum_{n=0}^{N} \frac{1}{n !}\left[\frac{z^{2}}{2 \sigma_{K}^{2}}\right]^{n} f(z) \\
& \leq \sum_{n=0}^{\infty} \frac{1}{n !}\left[\frac{z^{2}}{2 \sigma_{K}^{2}}\right]^{n} f(z)=\mathrm{e}^{z^{2} /\left(2 \sigma_{K}^{2}\right)} f(z),
\end{aligned}
$$

and so by (11) the validity of (4) will be guaranteed if

$$
\int_{-\infty}^{\infty} \mathrm{e}^{z^{2} /\left(2 \sigma_{K}^{2}\right)} f(z) \mathrm{d} z \equiv E\left\{\mathrm{e}^{Z^{2} /\left(2 \sigma_{K}^{2}\right)}\right\}<\infty
$$

Thus (12) is a sufficient condition for the validity of the expansion (4) in terms of the even moments of $Z$.

\section{Super- and Sub-Gaussian Distributions}

We have seen that the Laplace distribution gives a divergent expansion, and that (12) is a sufficient condition for validity of the expansion. We now put these results in the context of super- and sub-Gaussian distributions.

With respect to the GGD class of (5), distributions corresponding to the range $\alpha<2$ are more peaked and longer-tailed than the Gaussian and are often said to be 'super-Gaussian.' [2, p. 390],[25]. The Laplace distribution with $\alpha=1$ shown in Fig. 1(a) is such a super-Gaussian example. Those corresponding to $\alpha>2$ are flatter and shorter-tailed than the Gaussian and are called 'subGaussian.' [2, 25]. The uniform distribution $(\alpha=\infty)$ shown in Fig. 1(a) is such a sub-Gaussian example. 
Often the super/sub-Gaussian classification for distributions is made based on the kurtosis of the distribution $[10,14]$. The kurtosis, the fourth central moment divided by the variance squared, is given by $\beta_{2}=\Gamma(1 / \alpha) \Gamma(5 / \alpha) /\left[\Gamma^{2}(3 / \alpha)\right]$ for the GGD, depending only on $\alpha$. The kurtosis is 6,3 and 1.8 for the Laplace, Gaussian and uniform distributions. Under this classification a kurtosis exceeding 3 is identified with a super-Gaussian distribution, and less than 3 with a sub-Gaussian distribution.

While there is no doubt about the monotonic tail and peakedness behaviour of the distributions within the GGD class as $\alpha /$ kurtosis varies, and hence no doubt about consequent super- and sub-Gaussianity classifications, there is a real problem when using the kurtosis classification more widely. Fig. 1(b) shows Tukey's symmetric lambda distribution [12] when $\lambda=6$. The distribution has only a finite range. Its tail behaviour is uniform-like, while its central behaviour is Laplace-like. Its kurtosis is about 3.4 and hence it would be classified as super-Gaussian and yet has shorter tails than the Gaussian, which, based on the GGD class, we expect to be a sub-Gaussian characteristic! This illustrates that peakedness, kurtosis and tail length are not always synonymous, [12].

\subsection{Formal Definitions of Sub-Gaussianity}

A general definition of sub-Gaussianity is given in [13, 24]. A random variable $Z$ is said to be sub-Gaussian with parameter $\beta>0$, if for $u \in \mathbb{R}$, its moment generating function $M(u)$ satisfies

$$
M(u) \equiv E\left\{\mathrm{e}^{u Z}\right\} \leq \mathrm{e}^{u^{2} \beta^{2} / 2} .
$$

( $\beta$ is uniquely defined as the minimum of the values satisfying (13)). The mean must be zero [24]. We denote random variables of this class as being 'sGM.' Note a zero mean Gaussian variable would satisfy (13) with equality and $\beta^{2}=\sigma^{2}$ in this case. If $Z$ is bounded and has zero mean then $Z$ is sGM [24], so Tukey's lambda distribution of Fig. 1(b) is thus classified here as sub-Gaussian (unlike under kurtosis). 
Theorem 1. Let $Z$ be sGM. Let $\xi$ be any constant such that $\xi>2$. Then

$$
E\left\{\mathrm{e}^{Z^{2} /\left(\xi \beta^{2}\right)}\right\} \leq \frac{\xi+2}{\xi-2} .
$$

The proof can be found in A.1. In consideration of the sufficient condition (12), we thus have

$$
\int_{-\infty}^{\infty} \mathrm{e}^{z^{2} /\left(2 \sigma_{K}^{2}\right)} f(z) \mathrm{d} z \leq \int_{-\infty}^{\infty} \mathrm{e}^{z^{2} /\left(\xi \beta^{2}\right)} f(z) \mathrm{d} z \leq \frac{\xi+2}{\xi-2},
$$

for $\xi \beta^{2} \leq 2 \sigma_{K}^{2}$, i.e., $\beta^{2} \leq 2 \sigma_{K}^{2} / \xi<\sigma_{K}^{2}$. So we arrive at:

A sufficient condition for the validity of (4) is thus,

$$
Z \text { is sGM with } \beta<\sigma_{K} \text {. }
$$

This result can be further illustrated as follows. It is shown in [8, p. 200] that if $Z$ is sGM with parameter $\beta$ then

$$
E\left\{Z^{2 n}\right\} \leq 2^{n+1} n ! \beta^{2 n}, \quad n \geq 0 .
$$

So (3) gives

$$
\begin{aligned}
|V| & \leq \frac{1}{\sigma_{K} \sqrt{2 \pi}} \sum_{n=0}^{\infty}\left|\frac{(-1)^{n}}{2^{n} \sigma_{K}^{2 n} n !} E\left\{Z^{2 n}\right\}\right| \\
& =\frac{1}{\sigma_{K} \sqrt{2 \pi}} \sum_{n=0}^{\infty} \frac{1}{2^{n} \sigma_{K}^{2 n} n !} E\left\{Z^{2 n}\right\} \\
& \leq \frac{1}{\sigma_{K} \sqrt{2 \pi}} \sum_{n=0}^{\infty} \frac{1}{2^{n} \sigma_{K}^{2 n} n !} 2^{n+1} n ! \beta^{2 n} \\
& =\frac{2}{\sigma_{K} \sqrt{2 \pi}} \sum_{n=0}^{\infty}\left[\frac{\beta}{\sigma_{K}}\right]^{2 n}<\infty,
\end{aligned}
$$

provided $\beta<\sigma_{K}$.

Remark 1. It is worth stressing that (14) is a sufficient, but not necessary, condition. As an example, take the case of $Z$ being a uniformly distributed random variable over $[-b, b]$. This has variance $\sigma^{2}=b^{2} / 3$ and

$$
E\left\{Z^{2 n}\right\}=\frac{\left(3 \sigma^{2}\right)^{n}}{(2 n+1)},
$$


so that (3) gives

$$
V_{Z}=\frac{1}{\sigma_{K} \sqrt{2 \pi}} \sum_{n=0}^{\infty}\left(\frac{-3 \sigma^{2}}{2 \sigma_{K}^{2}}\right)^{n} \frac{1}{n !(2 n+1)},
$$

which is convergent for all finite, positive, $\sigma_{K}, \sigma$, (e.g., by the ratio test).

However, a uniform distribution on $[-b, b]$ is $\mathrm{sGM}$ with parameter $\beta=b \sqrt{ } 2=$ $\sigma \sqrt{ } 6$, [24], so that the sufficient condition $\beta<\sigma_{K}$ is equivalent to the requirement $\sigma \sqrt{ } 6<\sigma_{K}$, in contrast to our direct calculation finding that convergence holds for all finite, positive values $\sigma_{K}, \sigma$.

Remark 2. It is interesting to note that even with the definition of subGaussianity in (13), the tails of a probability density function $f_{Z}(\cdot)$ for a sGM variable need not be below that of a Gaussian distribution for all values of $|z|$ exceeding some value $z_{0}$; see [6].

An alternative definition of sub-Gaussianity was given in [22] based on the idea of relative convexity. Suppose we have a zero-mean random variable $Z$ with unimodal density $f(z) \propto \mathrm{e}^{-\psi(z)}$, with $\psi(z)$ symmetric and strictly increasing on $(0, \infty)$. Ordinarily, a function is said to be convex on an interval $I$ if it is true that, for $0 \leq \mu \leq 1$,

$$
\psi\left(\mu z_{0}+(1-\mu) z_{1}\right) \leq \mu \psi\left(z_{0}\right)+(1-\mu) \psi\left(z_{1}\right)
$$

for distinct $z_{0}, z_{1} \in I$, [3]. This means that for any two points $z_{0}, z_{1}$ in $I$, the function value at all intermediate points is no larger than the value of the linear function defined to match the value of $\psi$ at $z_{0}$ and $z_{1}$. Convexity can be extended to compare a function $\psi$ to non-linear functions. If the function $\psi$ is convex relative to some non-linear function $\varphi$, [22], write $\psi \succeq \varphi$. A particularly relevant case is when $\varphi(z)=z^{2}$; if $\psi \succeq \varphi$ then $\psi$ is said to be square-convex. In $[22] Z$ is said to be sub-Gaussian if

$$
\psi \text { is square-convex on }(0, \infty) \text {. }
$$

We denote random variables of this class as being 'sGC.' 
Theorem 2. Let $Z$ be sGC. Suppose

$$
\text { there exists } z_{0} \in(0, \infty) \text { such that } \frac{\psi^{\prime}\left(z_{0}\right)}{z_{0}}>\frac{1}{\sigma_{K}^{2}} \text {. }
$$

Then

$$
E\left\{\mathrm{e}^{Z^{2} /\left(2 \sigma_{K}^{2}\right)}\right\}<\infty,
$$

i.e., (12) and consequently the expansion (4) holds.

The proof can be found in A.2. As a consequence, when (18) is true, the expansion (4) is valid for sGC random variables.

Remark 3. The square convexity definition of sub-Gaussianity is almost equivalent to that given in [2], who defined sub-Gaussianity as holding when $\psi^{\prime}(z) / z$ is strictly increasing on $(0, \infty)$. We denote random variables of this class as being 'sGB.' For equivalence of sGC and sGB we need to require strict convexity - see A.3

Clearly the sGB class is a sub-class of sGC, since the stronger strict convexity is required.

Consider a random variable $Z$ with the generalized Gaussian distribution having $\alpha=2+\delta, \delta>0$. Then $\psi(z)=A(\alpha, \sigma) z^{2+\delta}$ for $z \in(0, \infty)$ and $\psi^{\prime}(z) / z=$ $A(\alpha, \sigma)(2+\delta) z^{\delta}$ which is strictly increasing on $(0, \infty)$, so $Z$ is sGB and hence sGC. (Also, $\psi^{\prime \prime}(z) / \psi^{\prime}(z)=(1+\delta) / z>1 / z$, and $\eta(z)$ is strictly convex on $(0, \infty)$; see A.3.)

Remark 4. In the case of strict convexity of $\psi(z)$ relative to $z^{2}$ we know from the defining criterion for $\mathrm{sGB}$ that $\psi^{\prime}(z) / z$, will be strictly increasing on $(0, \infty)$. Hence almost always there will exist a $z_{0}$ satisfying (18) and hence the expansion (4) will be valid.

\subsection{Discussion and Summary}

We have seen in this section that for distributions classified as sub-Gaussian via moment generating function, sGM, or via square-convexity, sGC, that conditions (14) and (18), respectively, are sufficient for the validity of the expansion of correntropy in terms of its even moments. These conditions involve the size of 

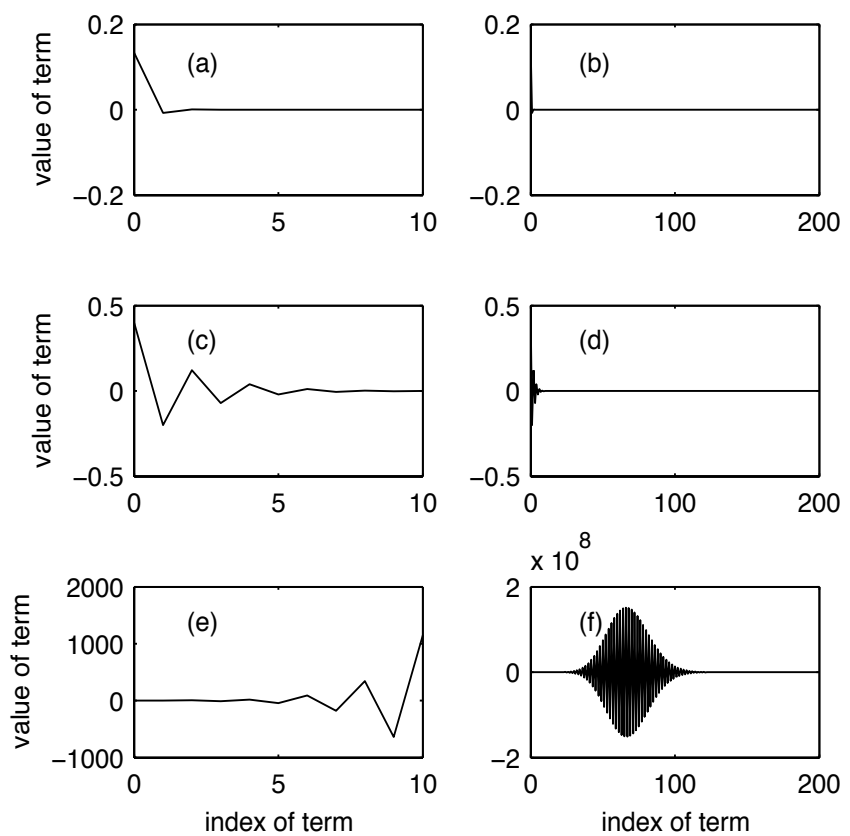

Figure 3: Terms in the expansion (4) for a GGD distribution $(\alpha=3)$ for $Z,(\sigma=1)$ and $\sigma_{K}=3$ for (a) and (b), $\sigma_{K}=1$ for (c) and (d), and $\sigma_{K}=0.5$ for (e) and (f).

the Gaussian kernel $\sigma_{K}$. It is difficult to make definitive statements for superGaussian distributions corresponding to $\psi^{\prime}(z) / z$ being strictly decreasing on $(0, \infty)$, [2], or $\psi$ being square-concave on $(0, \infty),[22]$. For example a random variable having the Laplace distribution, super-Gaussian under both definitions, gave rise to a divergent asymptotic series expansion (9). Under square-concavity, (rather than square-convexity), a typical inequality is in the wrong direction to be useful.

\subsection{Examples}

Here we consider two examples where the distributions are sGB and sGC (by the discussion following Remark 3). A convergent expansion (4) is expected by Remark 4. Consider the terms in expansion (4) for a GGD with $\alpha=3$ - see Fig. 3. For $\sigma_{K} / \sigma=3$, plots (a) and (b), and for $\sigma_{K} / \sigma=1$, plots (c) and (d), 

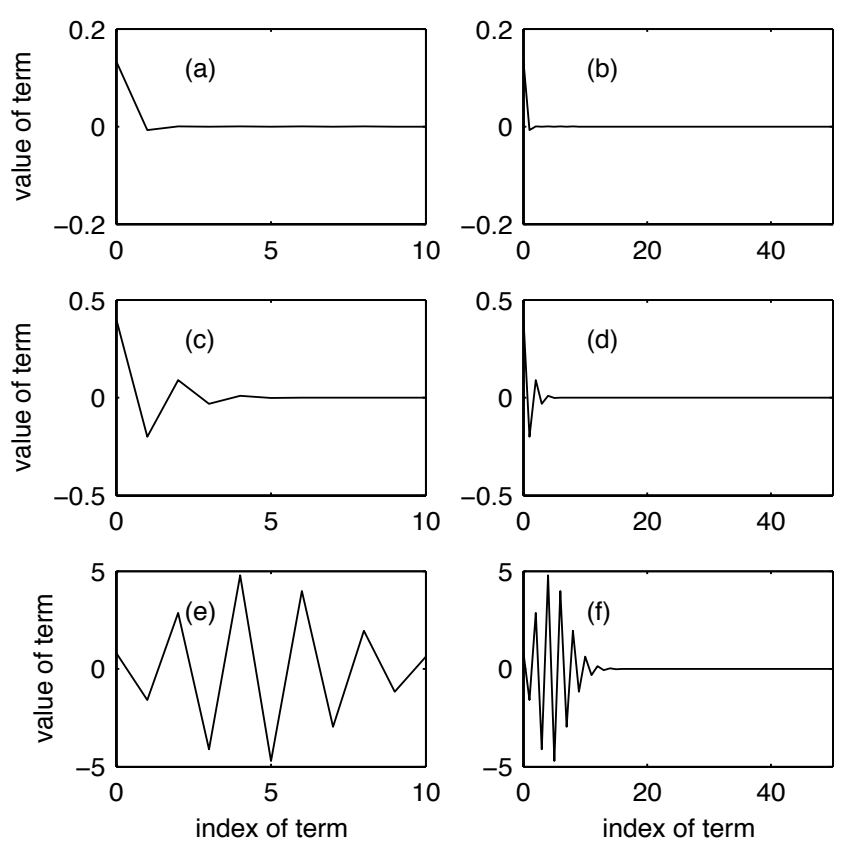

Figure 4: Terms in the expansion (4) for a uniform distribution for $Z,(\sigma=1)$ and $\sigma_{K}=3$ for (a) and (b), $\sigma_{K}=1$ for (c) and (d), and $\sigma_{K}=0.5$ for (e) and (f).

the terms in the series expansion quickly decay. The case $\sigma_{K} / \sigma=0.5$, plots (e) and (f), is interesting as we see that there is a large oscillation which is bounded but dies away. So in this case most of the contribution to correntropy would come from extremely high-order moments, not a desirable situation!

Fig. 4 examines the case $\alpha=\infty$, i.e., a uniform distribution. In this case the terms in series (4) die away quickly for $\sigma_{K} / \sigma=3,1$ and 0.5 .

These results are consistent with a convergent expansion in both cases. But we can see that the choice of $\sigma_{K}$ is quite crucial: choose it too large and correntropy will do no more than utilise second-order properties [11], but choose it too small and 'pathological' behaviour like that illustrated in Fig. 3(f) can arise. 


\section{Correntropy-based Blind Deconvolution}

\subsection{Preliminaries}

Given some observed series $\left\{X_{t}\right\}$ which is a distorted version of a source series $\left\{S_{t}\right\}$, and a blurring filter $\left\{h_{j}\right\}$, deconvolution is the attempt to remove the effect of the blurring filter to recover the original source (input) signal. When the blurring filter is assumed linear and translation-invariant but otherwise unknown, and knowledge of the source signal is limited to its statistical properties, the deconvolution is called 'blind.' Correntropy-based blind deconvolution was proposed in [23].

The mathematical model for the observed series $\left\{X_{t}\right\}$ is

$$
X_{t}=(h * S)_{t}+N_{t}=\sum_{j} h_{j} S_{t-j}+N_{t}
$$

where $\left\{N_{t}\right\}$ is assumed to be zero-mean Gaussian white noise that is independent of $\left\{S_{t}\right\}$ for all $t$. In practice we seek a deconvolver $\left\{w_{j}, j=0, \ldots, M-1\right\}$ for which the recovered series $\left\{Y_{t}\right\}$, given by [23],

$$
\hat{S}_{t}=Y_{t}=(w * X)_{t}=\sum_{j=0}^{M-1} w_{j} X_{t-j}=\boldsymbol{w}^{T} \boldsymbol{X}_{t},
$$

resembles as closely as possible the original series $\left\{S_{t}\right\}$. Here $\boldsymbol{w}=\left[w_{0}, \ldots, w_{M-1}\right]^{T}$ and $\boldsymbol{X}_{t}=\left[X_{t}, X_{t-1}, \ldots, X_{t-M+1}\right]^{T}$. The deconvolver $\left\{w_{t}\right\}$ is typically estimated iteratively by adjusting it until a chosen sample statistic of the deconvolved series, $\left\{Y_{t}\right\}=\left\{\hat{S}_{t}\right\}$ is close to that expected for $\left\{S_{t}\right\}$.

The source $\left\{S_{t}\right\}$ is often assumed to be independent and identically distributed (IID), so that the blurring process effectively Gaussianises the source [19]; most conventional blind deconvolution methods exploit this fact by using nonGaussianity as a deconvolution criterion. However the source may be coloured (correlated). In this case, if the colouring is known to be a linear translation-invariant filter, it can be removed by prewhitening when it is known a priori or treated as part of the blurring. Correntropy-based deconvolution was proposed as an alternative approach. 
In this context the correntropy function should be a function of lag $\tau$,

$$
V_{\tau} \equiv E\left\{K\left(Z_{t, \tau}\right)\right\}
$$

where $Z_{t, \tau}=S_{t}-S_{t-\tau}$. Santamaria et al. [23] suggested correntropy blind deconvolution should be carried out by minimizing the objective function or cost given by

$$
\sum_{\tau=1}^{P}\left[V_{\tau}-\hat{V}_{\tau}\right]^{2}
$$

where $P$ is the number of (non-zero) lags to use, $V_{\tau}$ is the true correntropy and $\hat{V}_{\tau}$ is the sample correntropy of the equalized/deconvolved series $\hat{Z}_{t, \tau}=Y_{t}-Y_{t-\tau}$, namely

$$
\hat{V}_{\tau}=\frac{1}{N-\tau} \sum_{t=\tau+1}^{N} K\left(\hat{Z}_{t, \tau}\right) .
$$

Since correntropy is sensitive to temporal correlation through time series lags, and distributional properties through the higher-order even moments of $\left\{Z_{t, \tau}\right\}$ - see (4) - it was advanced as a suitable objective function for deconvolution in [23].

We are most interested in the case where the source process $\left\{S_{t}\right\}$ is both nonGaussian and nonwhite (correlated) - since correntopy utilises both temporal and distributional properties - and the resulting correntropy is only a function of $\tau$ and not $t$. We would like more general series than filtered binary sequences used in [23]. We shall also be interested in the simpler case where $\left\{S_{t}\right\}$ is just nonGaussian and white.

\subsection{Stationarity of Source Process}

There are two fundamental cases to consider.

Suppose that $\left\{S_{t}\right\}$ is second-order stationary (SOS). Then $\left\{S_{t}-S_{t-\tau}\right\}$ is SOS also [21, p. 347], but $\left\{K\left(S_{t}-S_{t-\tau}\right)\right\}$, need not be SOS, since a zero-memory nonlinear transform of a SOS process yields a process that is not necessarily stationary (in any sense) [21, p. 307]. Hence the correntropy need not exist for SOS processes. 
Now suppose $\left\{S_{t}\right\}$ is strictly stationary (SS). Then $\left\{S_{t}-S_{t-\tau}\right\}$ is SS since a linear filtering of a SS process gives a SS process [21, p. 311] and $\left\{K\left(S_{t}-S_{t-\tau}\right)\right\}$ is also SS since a zero-memory nonlinear transform of a SS process yields a process that is also SS [21, p. 306]. (For example if $\left\{S_{t}\right\}$ is an IID sequence, then it is SS and hence so is $\left\{K\left(S_{t}-S_{t-\tau}\right)\right\}\left[5\right.$, p. 22].) Hence $V_{\tau}$ will depend only on $\tau$ as required.

\subsection{NonGaussianity of Source Process}

A suitable source process for correntropy, where the source process $\left\{S_{t}\right\}$ is strictly stationary and also both nonGaussian and nonwhite, is one generated by passing a SOS Gaussian process through a zero-memory nonlinearity (ZMNL). A Gaussian process $\left\{G_{t}\right\}$, say, is strictly stationary and if passed through a ZMNL

of the form $F^{-1} \Phi(\cdot)$, where $\Phi$ is the Gaussian distribution function and $F$ is a continuous strictly increasing distribution function for a particular marginal distribution for $\left\{S_{t}\right\}$, then $S_{t}=F^{-1} \Phi\left(G_{t}\right)$ will be strictly stationary with the specified marginal distribution [16].

For the case where the source process is merely strictly stationary and nonGaussian we can simply equate $\left\{S_{t}\right\}$ to an IID nonGaussian sequence.

We have thus established that source processes more general than filtered binary sequences exist satisfying the requirements for correntropy.

\subsection{Example Source Processes}

Suppose $\left\{S_{t}\right\}$ is an IID sequence of random variables, with standard deviation $\sigma_{S}$, each distributed with the Laplace distribution with $\operatorname{PDF} f_{S}(s)=$ $\left(1 /\left[\sigma_{S} \sqrt{2}\right]\right) \mathrm{e}^{-|s| \sqrt{2} / \sigma_{S}}$. Then $Z=S_{t}-S_{t-\tau}$ has PDF

$$
f(z)=\int_{-\infty}^{\infty} f_{S}(s) f_{S}(s-z) \mathrm{d} s,
$$

which reduces to the PDF

$$
f(z)=\frac{1}{2 \sigma_{S}^{2}} \mathrm{e}^{-|z| \sqrt{2} / \sigma_{S}}\left[|z|+\frac{\sigma_{S}}{\sqrt{2}}\right],
$$

which is symmetric about zero. Using this PDF, the even moments follow as

$$
E\left\{Z^{2 n}\right\}=\sigma_{S}^{2 n}(n+1) \Gamma(2 n+1) / 2^{n} .
$$


When $n=1$ we see that $E\left\{Z^{2}\right\} \equiv \sigma^{2}=2 \sigma_{S}^{2}$, as expected. For the terms of the sum (4) it is found that $\left|u_{n+1} / u_{n}\right|=\left[(2 n+1)(n+2) \sigma^{2}\right] /\left[4(n+1) \sigma_{K}^{2}\right] \rightarrow \infty$ as $n \rightarrow \infty$, so the sum (4) is divergent by the ratio test.

Now let $\left\{S_{t}\right\}$ be an IID sequence of random variables, each distributed uniformly over $[-b, b]$. For such a distribution $\sigma_{S}^{2}=b^{2} / 3$. Then its moment generating function is $M_{S}(u)=\left(\mathrm{e}^{b u}-\mathrm{e}^{-b u}\right) /(2 b u)$. Then $Z=S_{t}-S_{t-\tau}$ has a moment generating function

$$
M(u)=M_{S}(u) M_{S}(-u)=\frac{\mathrm{e}^{2 b u}+\mathrm{e}^{-2 b u}-2}{(2 b u)^{2}}=\frac{\sinh ^{2}(b u)}{(b u)^{2}},
$$

which identifies $Z$ as having a triangular distribution centered around zero with range $[-2 b, 2 b]$ and

$$
E\left\{Z^{2 n}\right\}=\frac{(2 b)^{2 n}}{(2 n+1)(n+1)} .
$$

When $n=1, E\left\{Z^{2}\right\} \equiv \sigma^{2}=2 b^{2} / 3=2 \sigma_{S}^{2}$, as expected. Since $\left|u_{n+1} / u_{n}\right|=$ $\left[(2 b)^{2}(2 n+1)\right] /\left[2 \sigma_{K}^{2}(2 n+3)(n+2)\right] \rightarrow 0$ as $n \rightarrow \infty$, the sum (4) is convergent by the ratio test for all choices of $b$. As already mentioned below (13) since $Z$ is bounded and has zero mean then $Z$ is sGM. Take the case $b=0.5$, for which $\sigma=1 / \sqrt{ } 6 \approx 0.41 ;$ numerical computations show that in this case $\beta$ in (13) is approximately 0.41 . With the sufficient condition (14) for the convergence of (4) in mind, the choices $\sigma_{K} / \sigma=3,1$ and 0.5 correspond to $\beta<\sigma_{K}, \beta \approx \sigma_{K}$ and $\beta>\sigma_{K}$. But of course we know (4) is convergent here. We consider the expansion (4) for this triangular distribution in Fig. 5. For $\sigma_{K} / \sigma=3$, plots (a) and (b), and for $\sigma_{K} / \sigma=1$, plots (c) and (d), the terms in the series expansion quickly decay. For $\sigma_{K} / \sigma=0.5$, plots (e) and (f), the same sort of pathological behaviour that we observed in Fig. 3(e) and (f) again arises. Correntropy has become dominated by very high-order moments by simply halving $\sigma_{K}$. It is intriguing to think that the violation of the sufficient condition $\beta<\sigma_{K}$ in this latter case might be linked to the pathological convergence behaviour that we observe. 

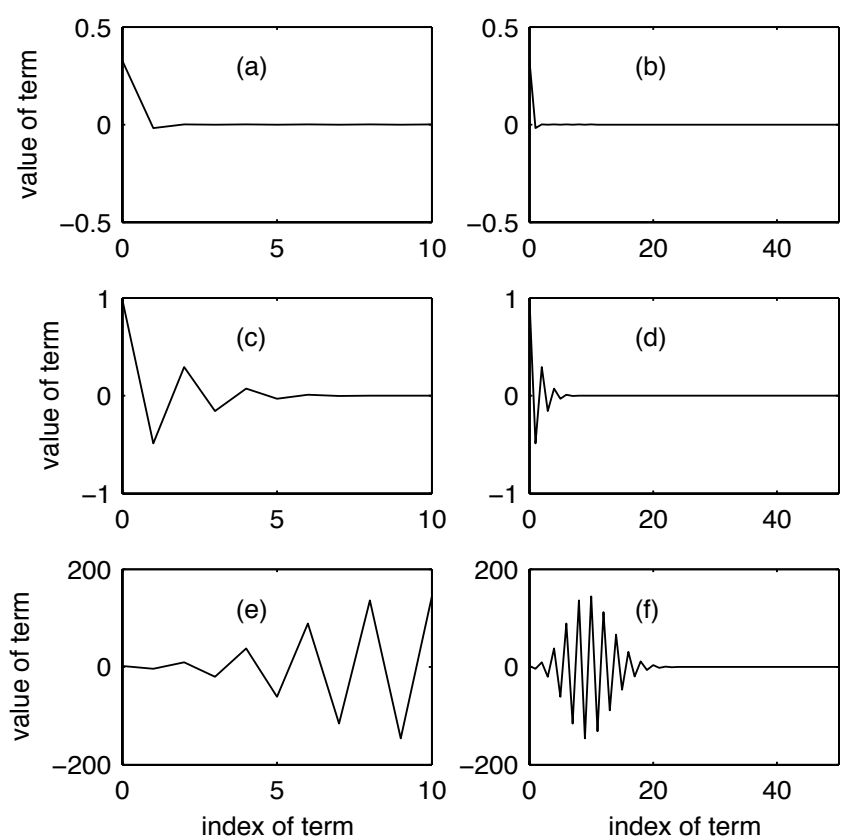

Figure 5: Terms in the expansion (4) for a triangular distribution for $Z,(\sigma=1 / \sqrt{ } 6)$, and $\sigma_{K} / \sigma=3$ for (a) and (b), $\sigma_{K} / \sigma=1$ for (c) and (d), and $\sigma_{K} / \sigma=0.5$ for (e) and (f).

\subsection{The Cost Surface}

We now consider the solutions corresponding to the minimization of the objective/cost function (21) in the noise-free case. Now $\left\{X_{t}\right\}$ is stationary, $\left(S_{t}\right.$ is strictly stationary), and (nearly) Gaussian due to the effects of the convolution [19]. Now $Y_{t}=w_{0} X_{t}+w_{1} X_{t-1}+\cdots+w_{M-1} X_{t-M+1}$ and let $Y_{t}^{\prime}=w_{M-1} X_{t}+$ $w_{M-2} X_{t-1}+\cdots+w_{0} X_{t-M+1}$. Then, with $\hat{Z}_{t, \tau}=Y_{t}-Y_{t-\tau}$ and $\hat{Z}_{t, \tau}^{\prime}=Y_{t}^{\prime}-Y_{t-\tau}^{\prime}$,

$$
\begin{array}{rll}
\hat{Z}_{t, \tau} & =w_{0}\left(X_{t}-X_{t-\tau}\right)+w_{1}\left(X_{t-1}-X_{t-\tau-1}\right) \\
& \cdots & +w_{M-1}\left(X_{t-M+1}-X_{t-M+1-\tau}\right) \\
\hat{Z}_{t, \tau}^{\prime} & = & w_{M-1}\left(X_{t}-X_{t-\tau}\right)+w_{M-2}\left(X_{t-1}-X_{t-\tau-1}\right) \\
& \cdots & +w_{0}\left(X_{t-M+1}-X_{t-M+1-\tau}\right) .
\end{array}
$$

Under Gaussianity, the joint distributions of $X_{t}, X_{t-1}, \ldots, X_{t-M+1}$ and $X_{t-M+1}$, $X_{t-M+2}, \ldots, X_{t}$ are identical, so that $X_{t}-X_{t-\tau}$ and $X_{t-M+1}-X_{t-M+1-\tau}$ are 
exchangeable, (their statistical structure is invariant to an exchange), $X_{t-1}-$ $X_{t-\tau-1}$ and $X_{t-M+2}-X_{t-M+2-\tau}$, are exchangeable, etc. Hence, the correntropy computed from $\left\{\hat{Z}_{t, \tau}\right\}$ and $\left\{\hat{Z}_{t, \tau}^{\prime}\right\}$ will be the same, and, asymptotically, so will be the expected value of the correntropy objective function (see A.4 for details). There will therefore not only be two 'optimal' solutions, $\boldsymbol{w}^{(1)}=\left( \pm w_{0}, \pm w_{1}, \ldots, \pm w_{M-1}\right)$, (the sign uncertainty in a blind deconvolution is a well-known effect and must be resolved by physical considerations), but also the two 'sub-optimal' solutions $\boldsymbol{w}^{(2)}=\left( \pm w_{M-1}, \pm w_{M-2}, \ldots, \pm w_{0}\right)$. Suboptimal solutions have also been observed in [20], but no explanation or analysis was given.

As illustration we consider a blurring filter of the form $h_{j}=1 / 2^{j}, j=$ $0, \ldots, 20$. If $\left\{h_{j}\right\}$ was not truncated at $j=20$ but was of infinite length the exact inverse filter would be $(1,-1 / 2)$. Hence the optimal deconvolution solutions are, to a close approximation, $\boldsymbol{w}^{(1)}=( \pm 1, \mp 1 / 2)$. (The solution $(-1,+1 / 2)$ takes into account the implicit sign uncertainty in deconvolution.) We expect other solutions at $\boldsymbol{w}^{(2)}=( \pm 1 / 2, \mp 1)$, corresponding to the interchange of $w_{0}$ and $w_{1}$. Fig. 6 shows the south-east quadrant of the logarithm of the estimated correntropy cost (21) with respect to the deconvolver when the source $\left\{S_{t}\right\}$ has a Gaussian distribution. Results are shown for four values of the ratio $\sigma_{K} / \sigma$, namely $5,3,1$ and 0.5 . The optimal, $(+1,-1 / 2)$, and predicted sub-optimal solution, $(+1 / 2,-1)$, in this south-east quadrant are marked by plus signs. The optimal solution and the sub-optimal solution agree very closely with the minima of the cost surface for all values of $\sigma_{K} / \sigma$, as predicted by the theory presented above.

Fig. 7 shows the same sort of plots, but this time for a Laplace-distributed source. The optimal solution $(+1,-1 / 2)$ agrees closely with the minimum of the cost surface for all values of $\sigma_{K} / \sigma$, while the other minimum of the cost surface converges to the predicted sub-optimal solution $(1 / 2,-1)$ as $\sigma_{K} / \sigma$ increases. Fig. 8 also shows the same sort of plots, but this time for a uniformly-distributed source. For the super-Gaussian source the convergence of the minimum of the surface to the sub-optimal solution $(1 / 2,-1)$ is clockwise, while for the sub- 

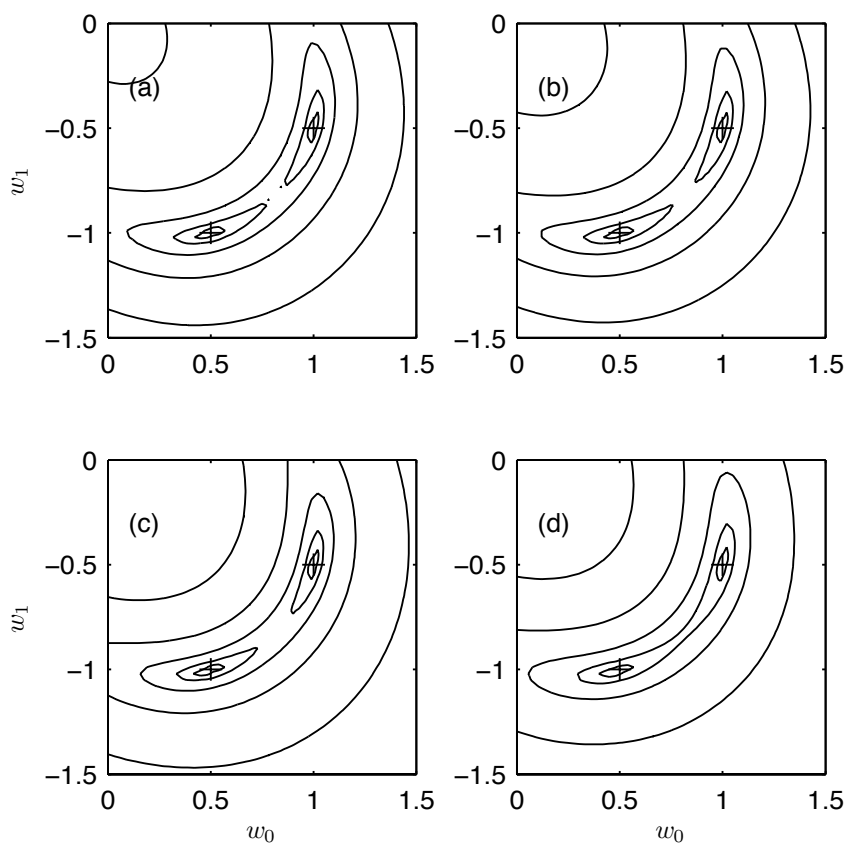

Figure 6: Estimated log cost of the correntropy algorithm for Gaussian distribution for $\left\{S_{t}\right\}$ and (a) $\sigma_{K} / \sigma=5$, (b) $\sigma_{K} / \sigma=3$, (c) $\sigma_{K} / \sigma=1$, and (d) $\sigma_{K} / \sigma=0.5$, with respect to $\boldsymbol{w}=\left(w_{0}, w_{1}\right)^{T} . N=15000$ and $P=4$.

Gaussian source the convergence is counter-clockwise.

Thus increasing $\sigma_{K} / \sigma$ for a given distribution of $\left\{S_{t}\right\}$ has a similar effect on the sub-optimal minima of the cost surface as increasing the Gaussianity of $\left\{S_{t}\right\}$ for a given $\sigma_{K} / \sigma$ : in both cases the sub-optimal minima of the cost surface become coincident with the sub-optimal solution $\boldsymbol{w}^{(2)}$ predicted by the Gaussian theory.

\subsection{Further comments on results}

In the experiments of Section 4.5, $\left\{S_{t}\right\}$ was IID, whatever its distribution. Hence $Z=Z_{t, \tau}=S_{t}-S_{t-\tau}$ does not depend on $t$ or $\tau$ and each $V_{\tau}$, (of the form (20)), in (21) is the same for a given distribution of $\left\{S_{t}\right\}$. On the other hand $\hat{Z}_{t, \tau}=Y_{t}-Y_{t-\tau}=\hat{S}_{t}-\hat{S}_{t-\tau}$ and hence $\hat{V}_{\tau}$ will vary with $\tau$ because the differences are of correlated random variables. The correlation of $Y_{t}=\hat{S}_{t}$ will 

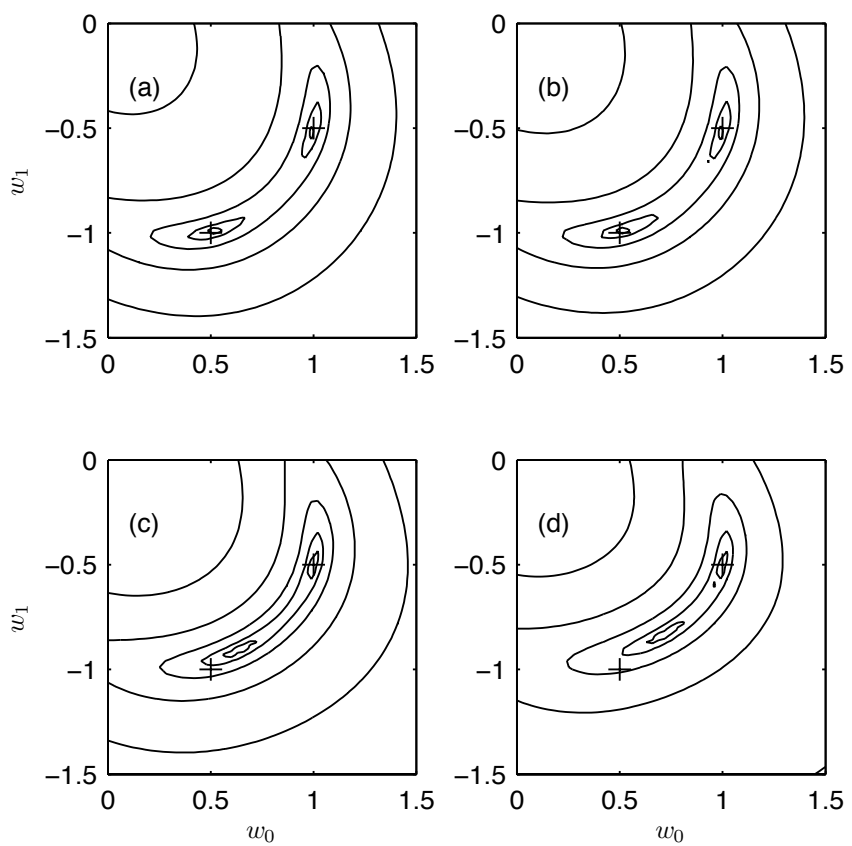

Figure 7: Estimated log cost of the correntropy algorithm for Laplace distribution for $\left\{S_{t}\right\}$ and (a) $\sigma_{K} / \sigma=5$, (b) $\sigma_{K} / \sigma=3$, (c) $\sigma_{K} / \sigma=1$, and (d) $\sigma_{K} / \sigma=0.5$, with respect to $\boldsymbol{w}=\left(w_{0}, w_{1}\right)^{T} . N=15000$ and $P=4$.

depend on the blurring filter and the deconvolution filter and will be least when the deconvolution filter is optimal, in which case the objective/cost function (21) will be minimized. There is a minimum in the cost surface at the location of the optimal deconvolver $(1,-1 / 2)$ in Figs. 6,7 and 8 for Gaussian, superand sub-Gaussian $\left\{S_{t}\right\}$, the latter two distributions giving rise to pathological behaviour of (4) (uniform distribution) and a divergent expansion for (4) (Laplace distribution), as shown in Section 4.4. It thus seems that pathological behaviour or divergence of (4) need not prevent successful correntropy-based deconvolution in the sense of the coincidence of a cost surface minimum with the optimal deconvolver, but also that other minima of the correntropy cost surface are sensitive to the type of nonGaussianity and the size of $\sigma_{K}$. 

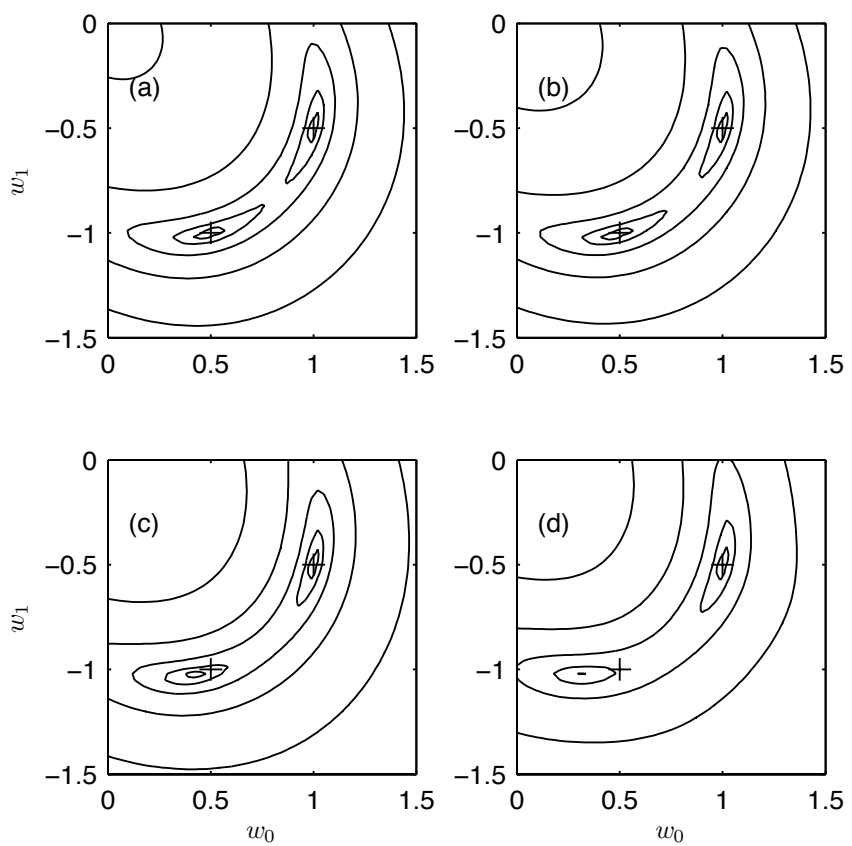

Figure 8: Estimated log cost of the correntropy algorithm for uniform distribution for $\left\{S_{t}\right\}$ and (a) $\sigma_{K} / \sigma=5$, (b) $\sigma_{K} / \sigma=3$, (c) $\sigma_{K} / \sigma=1$, and (d) $\sigma_{K} / \sigma=0.5$, with respect to $\boldsymbol{w}=\left(w_{0}, w_{1}\right)^{T} . N=15000$ and $P=4$.

\section{Concluding Remarks}

The correntropy function is an interesting and useful similarity measure which has been interpreted as involving the higher-order even moments of $Z=$ $X-Y$. We have looked in some detail at the effect the ratio $\sigma_{K} / \sigma$ and the distribution of $Z$ have on this interpretation. We demonstrated in Section 3 that the expansion of correntropy, and its interpretation, in terms of even moments of $Z$, can be guaranteed when $Z$ is sub-Gaussian according to the definition (13) and the condition $\beta<\sigma_{K}$ holds, or is sub-Gaussian according to the definition (17) and condition (18) holds. The Laplace distribution for $Z$ (super-Gaussian under the kurtosis or square-concave classifications) gave rise to an asymptotic series expansion corresponding to (4) being divergent. We reiterate that it is not necessary for the expansion (4) to exist for correntropy to exist. However the 
perception that "The use of higher-order moment information in correntropy is controlled smoothly by the kernel size ..." ([17, p. 5297]) appears to be a rather over-simplified view of the situation.

In the blind deconvolution setting it was illustrated that successful correntropybased deconvolution - in the sense of the coincidence of a cost surface minimum with the optimal deconvolver - can be achieved for IID sources $\left\{S_{t}\right\}$ ranging through super-Gaussian to sub-Gaussian. As $\sigma_{K}$ becomes larger, the suboptimal minima of the correntropy cost surface are pulled towards the theoretical sub-optimal solutions of the deconvolution problem predicted under Gaussianity. Clearly the choice of $\sigma_{K}$ is fundamental and more research is needed to appreciate the practical utility of correntropy within the various settings, such as seismic signal processing and communications, where deconvolution is ubiquitous.

\section{Acknowledgements}

The authors thank the reviewers for helpful comments which led to an improved exposition. Zi Yang thanks the EPSRC (UK) for financial support.

\section{A. Proofs}

\section{A.1. Proof of Theorem 1}

Firstly we note that

$$
\begin{aligned}
\int_{0}^{\infty} g^{\prime}(z) P(Z>z) \mathrm{d} z & =\int_{z=0}^{\infty} g^{\prime}(z)\left[\int_{y=z}^{\infty} f(y) \mathrm{d} y\right] \mathrm{d} z \\
& =\int_{y=0}^{\infty} f(y)\left[\int_{z=0}^{y} g^{\prime}(z) \mathrm{d} z\right] \mathrm{d} y \\
& =\int_{0}^{\infty}[g(y)-g(0)] f(y) \mathrm{d} y \\
& =\int_{0}^{\infty} g(y) f(y) \mathrm{d} y \\
& -g(0) \int_{0}^{\infty} f(y) \mathrm{d} y
\end{aligned}
$$


i.e.,

$$
\begin{aligned}
\int_{0}^{\infty} g(y) f(y) \mathrm{d} y & =\int_{0}^{\infty} g^{\prime}(z) P(Z>z) \mathrm{d} z \\
& +g(0) \int_{0}^{\infty} f(y) \mathrm{d} y .
\end{aligned}
$$

Similarly,

$$
\begin{aligned}
\int_{-\infty}^{0} g(y) f(y) \mathrm{d} y= & -\int_{-\infty}^{0} g^{\prime}(z) P(Z<z) \mathrm{d} z \\
& +g(0) \int_{-\infty}^{0} f(y) \mathrm{d} y .
\end{aligned}
$$

So

$$
\begin{aligned}
\int_{-\infty}^{\infty} g(y) f(y) \mathrm{d} y & =\int_{0}^{\infty} g^{\prime}(z) P(Z>z) \mathrm{d} z \\
& -\int_{-\infty}^{0} g^{\prime}(z) P(Z<z) \mathrm{d} z+g(0) .
\end{aligned}
$$

Let $g(z) \equiv \mathrm{e}^{z^{2} /\left(\xi \beta^{2}\right)}$. So $g^{\prime}(z)=\left[2 z /\left(\xi \beta^{2}\right)\right] \exp \left[z^{2} /\left(\xi \beta^{2}\right)\right]$ and $g(0)=1$. Then (23) gives

$$
\begin{aligned}
E\left\{\mathrm{e}^{Z^{2} /\left(\xi \beta^{2}\right)}\right\}= & \int_{0}^{\infty} \frac{2 z}{\xi \beta^{2}} \mathrm{e}^{z^{2} /\left(\xi \beta^{2}\right)} P(Z>z) \mathrm{d} z \\
& -\int_{-\infty}^{0} \frac{2 z}{\xi \beta^{2}} \mathrm{e}^{z^{2} /\left(\xi \beta^{2}\right)} P(Z<z) \mathrm{d} z+1 .
\end{aligned}
$$

Making the change of variable $z \rightarrow-z$ in the second integral on the right we get

$$
\begin{aligned}
E\left\{\mathrm{e}^{Z^{2} /\left(\xi \beta^{2}\right)}\right\}= & \int_{0}^{\infty} \frac{2 z}{\xi \beta^{2}} \mathrm{e}^{z^{2} /\left(\xi \beta^{2}\right)} P(Z>z) \mathrm{d} z \\
& +\int_{0}^{\infty} \frac{2 z}{\xi \beta^{2}} \mathrm{e}^{z^{2} /\left(\xi \beta^{2}\right)} P(Z<-z) \mathrm{d} z+1 .
\end{aligned}
$$

But since $Z$ is sub-Gaussian we know, [24], that $P(Z>z) \leq \mathrm{e}^{-z^{2} /\left(2 \beta^{2}\right)}$ and moreover, since $-Z$ is also sub-Gaussian with parameter $\beta,[24], P(Z<-z) \leq$ $\mathrm{e}^{-z^{2} /\left(2 \beta^{2}\right)}$ also. Hence,

$$
E\left\{\mathrm{e}^{Z^{2} /\left(\xi \beta^{2}\right)}\right\} \leq \frac{4}{\xi \beta^{2}} \int_{0}^{\infty} z \mathrm{e}^{-z^{2}\left[\frac{1}{2}-\frac{1}{\xi}\right] / \beta^{2}} \mathrm{~d} z+1 .
$$


But for $p>0$,

$$
\int_{0}^{\infty} z \mathrm{e}^{-p z^{2}} \mathrm{~d} z=(2 p)^{-1}
$$

So (24) gives

$$
E\left\{\mathrm{e}^{Z^{2} /\left(\xi \beta^{2}\right)}\right\} \leq \frac{4}{\xi-2}+1=\frac{\xi+2}{\xi-2},
$$

which completes the proof.

\section{A.2. Proof of Theorem 2}

Suppose there exists a point $z_{0} \in(0, \infty)$ such that $\psi^{\prime}\left(z_{0}\right) / z_{0}>1 / \sigma_{K}^{2}$. Since $\psi(z)$ is square-convex we know [22] that for $z \in(0, \infty)$,

$$
\psi\left(z_{0}\right)-\psi(z) \leq \frac{\psi^{\prime}\left(z_{0}\right)}{2 z_{0}}\left[z_{0}^{2}-z^{2}\right] .
$$

Hence,

$$
\mathrm{e}^{-\psi(z)} \leq C\left(z_{0}\right) \mathrm{e}^{-\frac{\psi^{\prime}\left(z_{0}\right)}{2 z_{0}} z^{2}},
$$

where $C\left(z_{0}\right)=\exp \left(\left(z_{0} / 2\right) \psi^{\prime}\left(z_{0}\right)-\psi\left(z_{0}\right)\right)$. Then, with $D$ a constant of proportionality,

$$
\begin{aligned}
E\left\{\mathrm{e}^{Z^{2} /\left(2 \sigma_{K}^{2}\right)}\right\} & =\int_{-\infty}^{\infty} \mathrm{e}^{z^{2} /\left(2 \sigma_{K}^{2}\right)} f(z) \mathrm{d} z \\
& =D \int_{-\infty}^{\infty} \mathrm{e}^{z^{2} /\left(2 \sigma_{K}^{2}\right)} \mathrm{e}^{-\psi(z)} \mathrm{d} z \\
& \leq C\left(z_{0}\right) D \int_{-\infty}^{\infty} \mathrm{e}^{z^{2} /\left(2 \sigma_{K}^{2}\right)} \mathrm{e}^{-z^{2} \psi^{\prime}\left(z_{0}\right) /\left(2 z_{0}\right)} \mathrm{d} z \\
& =C\left(z_{0}\right) D \int_{-\infty}^{\infty} \mathrm{e}^{-z^{2}\left[\frac{\psi^{\prime}\left(z_{0}\right)}{z_{0}}-\frac{1}{\sigma_{K}^{2}}\right] / 2} \mathrm{~d} z<\infty
\end{aligned}
$$

under the assumption that $\psi^{\prime}\left(z_{0}\right) / z_{0}>1 / \sigma_{K}^{2}$.

\section{A.3. Proof of Remark 3}

A function is said to be strictly convex on an interval $I$ if it is true that, for $0<\mu<1$,

$$
\psi\left(\mu z_{0}+(1-\mu) z_{1}\right)<\mu \psi\left(z_{0}\right)+(1-\mu) \psi\left(z_{1}\right),
$$


for distinct $z_{0}, z_{1} \in I$, [4]. Write $\mathrm{e}^{-\psi(z)}=\mathrm{e}^{-\eta\left(z^{2}\right)}=\mathrm{e}^{-\eta(\varphi(z))}$, with $\varphi(z)=z^{2}$, also a strictly convex function. Then

$$
\psi(z)=\eta(\varphi(z)) \Rightarrow\left(\psi \circ \varphi^{-1}\right)(z)=\eta(z) .
$$

But $\psi$ is strictly convex relative to $\varphi$ on $(0, \infty)$, if $\psi \circ \varphi^{-1}$ is strictly convex on $(0, \infty)$, (by extension of $[22$, p. 43$]$ to strict convexity); hence $\eta$ will be strictly convex on $(0, \infty)$. Now $\eta^{\prime}\left(z^{2}\right)=\psi^{\prime}(z) /(2 z)$. Since $\eta$ is strictly convex, $\eta^{\prime}(z)$ is strictly increasing with $z \in(0, \infty)$, (by extension of [3, p. 117]), and hence so is $\eta^{\prime}\left(z^{2}\right)$ and $\psi^{\prime}(z) / z$, and so $Z$ is sGB, i.e., sub-Gaussian by the definition of [2]. Here strict convexity of $\eta$ implies that $\psi^{\prime}(z) / z$ is strictly increasing; however the converse is also true [3, p. 117]), and so the definitions of [22] and [2], i.e., sGC and $\mathrm{sGB}$, are equivalent when $\psi(z)$ is strictly convex relative to $\varphi(z)=z^{2}$ on $(0, \infty)$. Strict convexity can be partially characterized by secondorder conditions [4, p. 71]: if

$$
\frac{\mathrm{d}^{2}}{\mathrm{~d} z^{2}}\left(\psi \circ \varphi^{-1}\right)(z)>0, \quad z \in(0, \infty)
$$

then $\eta(z)=\left(\psi \circ \varphi^{-1}\right)(z)$ is strictly convex. This is equivalent to [22, p. 43]

$$
\frac{\psi^{\prime \prime}(z)}{\psi^{\prime}(z)}>\frac{\varphi^{\prime \prime}(z)}{\varphi^{\prime}(z)}=\frac{1}{z} .
$$

So if (27) holds, $\eta(z)=\left(\psi \circ \varphi^{-1}\right)(z)$ is strictly convex.

\section{A.4. Existence of Unwanted Minima}

For clarity here we restore the subscript on $V$ indicating the random variable under consideration. In the limit that $N \rightarrow \infty$, the expected value of the cost surface (objective function) in (21) can be written as:

$$
\begin{array}{rl}
\sum_{\tau=1}^{P} & E\left\{\left[\hat{V}_{\hat{Z}, \tau}-V_{Z, \tau}\right]^{2}\right\} \\
& =\sum_{\tau} E\left[\hat{V}_{\hat{Z}, \tau}-E\left\{\hat{V}_{\hat{Z}, \tau}\right\}\right]^{2}+\sum_{\tau}\left[E\left\{\hat{V}_{\hat{Z}, \tau}\right\}-V_{Z, \tau}\right]^{2} \\
& =\sum_{\tau} \operatorname{var}\left\{\hat{V}_{\hat{Z}, \tau}\right\}+\sum_{\tau}\left[V_{\hat{Z}, \tau}-V_{Z, \tau}\right]^{2} \\
& \rightarrow \sum_{\tau}\left[V_{\hat{Z}, \tau}-V_{Z, \tau}\right]^{2},
\end{array}
$$


as the sample correntropy is an unbiased and consistent estimator.

Therefore, if there exists $\boldsymbol{w}^{(1)}$ and $\boldsymbol{w}^{(2)}$ such that the correntropy function $V_{\hat{Z}, \tau}$ is the same, then the expected value of the cost surface at these two points would also be identical as $N \rightarrow \infty$.

\section{References}

[1] M. Abramowitz and I. Stegun, Handbook of Mathematical Functions. New York: Dover, 1965.

[2] A. Benveniste, M. Goursat \& G. Ruget, "Robust identification of a nonminimum phase system: blind adjustment of a linear equalizer in data communications," IEEE Trans. Automatic Control, vol. 25, 385-99, 1980.

[3] K. G. Binmore, Mathematical Analysis, 2nd. Edition. Cambridge, UK: Cambridge University Press, 1982.

[4] S. Boyd \& L. Vandenberghe, Convex Optimization. Cambridge, UK: Cambridge University Press, 2004.

[5] D. R. Brillinger, Time Series: Data Analysis and Theory. New York: McGraw-Hill, 1981.

[6] V. V. Buldygin \& Yu. V. Kozachenko, "Sub-Gaussian random variables," Ukrainian Math. J., vol. 32, pp. 483-9, 1980.

[7] R. B. Dingle, Asymptotic Expansions: Their Derivation and Interpretation. New York: Academic Press, 1973.

[8] D. J. H. Garling, Inequalities: a Journey into Linear Analysis. Cambridge, UK: Cambridge University Press, 2007.

[9] A. Gunduz \& J. C. Principe, "Correntropy as a novel measure for nonlinearity tests," Signal Processing, vol. 89, pp. 14-23, 2009. 
[10] B.-C. Ihm, D.-J. Park \& Y.-H. Kwon, "Blind separation for mixtures of sub-Gaussian and super-Gaussian sources," In Proc. IEEE Int. Symposium on Circuits and Systems, Geneva, May 28-3-1, vol. III, pp. 738-741, 2000.

[11] K.-H. Jeong, W. Liu, S. Han., E. Hasanbelliu \& J. C. Principe, "The correntropy MACE filter," Pattern Recognition, vol. 42, 871-85, 2009.

[12] B. L. Joiner \& J. R. Rosenblatt, "Some properties of the range in samples from Tukey's symmetric lambda distributions," J. American Statistical Association, vol. 66, 394-9, 1971.

[13] J. P. Kahane, "Local properties of functions in terms of random Fourier series," Studia Mathematica, vol. 19, pp. 1-25, 1960.

[14] T.-W. Lee \& T. J. Sejnowski, "Independent component analysis for mixed sub-Gaussian and super-Gaussian sources," In Proc. 4th Joint Symposium on Neural Computation, Institute for Neural Computation, UCSD, 7, pp. 132-40, 1997.

[15] R. Li, W. Liu \& J. C. Principe, "A unifying criterion for instantaneous blind source separation based on correntropy," Signal Processing, vol. 87, 1872-81.

[16] B. Liu \& D. C. Munson, "Generation of a random sequence having a jointly specified marginal distribution and autocovariance," IEEE Trans. Acoustics, Speech, and Signal Process., vol. 30(6), pp. 973-83, 1982.

[17] W. Liu, P. P. Pokharel and J. C. Principe, "Correntropy: properties and applications in non-Gaussian signal processing," IEEE Trans. Signal Process., vol. 55, pp. 5286-98, 2007.

[18] S. Mallat, A Wavelet Tour of Signal Processing. San Diego: Academic Press, 1998.

[19] C. L. Mallows, "Linear processes are nearly Gaussian," Journal of Applied Probability, vol. 4, pp. 313-29, 1967. 
[20] A. Neves, C. Wada, R. Suyama, R. Attux, J. M. T. Romano, An analysis of unsupervised signal processing methods in the context of correlated sources, in: T. Adali, C. Jutten, J. M. T. Romano, A. K. Barros (Eds.), Independent Component Analysis and Signal Separation, Lecture Notes in Computer Science 5441, Springer, 2009.

[21] A. Papoulis, Probability, Random Variables, and Stochastic Processes. New York: McGraw-Hill, 1972.

[22] J. A. Palmer \& K. Kreutz-Delgado, "A general framework for component estimation," Proc. 4th Int. Symp. on Independent Component Analysis and Blind Signal Separation, Nara, Japa, April 1-4, pp. 41-6, 2003.

[23] I. Santamaría, P. P. Pokharel \& J. P. Principe, "Generalized correlation function: definition, properties, and application to blind equalization," IEEE Trans. Signal Process., vol. 54(6), pp. 2187-97, 2006.

[24] R. L. Taylor \& T.-C. Hu, "Sub-Gaussian techniques in proving strong laws of large numbers," The American Mathematical Monthly, vol. 94, 295-9, 1987.

[25] A. T. Walden, "A comparison of stochastic gradient and MED-type deconvolution algorithms," Signal Processing, vol. 15, 203-11, 1988. 